\title{
Study of the $D_{s}^{+} \rightarrow a_{0}(980) \rho$ and $a_{0}(980) \omega$ decays
}

\author{
Yao Yu ${ }^{1, a}$, Yu-Kuo Hsiao ${ }^{2, b}$, Bai-Cian Ke ${ }^{3, c}$ \\ ${ }^{1}$ Chongqing University of Posts and Telecommunications, Chongqing 400065, China \\ ${ }^{2}$ School of Physics and Information Engineering, Shanxi Normal University, Linfen 041004, China \\ ${ }^{3}$ School of Physics and Microelectronics, Zhengzhou University, Zhengzhou 450001, Henan, China
}

\begin{abstract}
We study $D_{s}^{+} \rightarrow \rho^{0(+)} a_{0}^{+(0)}, D_{s}^{+} \rightarrow \omega a_{0}^{+}$, and the resonant $D_{s}^{+} \rightarrow \rho a_{0}, a_{0} \rightarrow \eta \pi(K K)$ decays. In the final state interaction, where $D_{s}^{+} \rightarrow\left(\eta^{(\prime)} \pi^{+}, K^{+} \bar{K}^{0}\right)$ are followed by the $\left(\eta^{(\prime)} \pi^{+}, K^{+} \bar{K}^{0}\right)$ to $\rho^{0(+)} a_{0}^{+(0)}$ rescatterings, we predict $\mathcal{B}\left(D_{s}^{+} \rightarrow \rho^{0(+)} a_{0}^{+(0)}\right)=(3.0 \pm 0.3 \pm 1.0) \times 10^{-3}$. Due to the cancellation of the rescattering effects and the suppressed short-distance $W$ annihilation contribution, we expect that $\mathcal{B}\left(D_{s}^{+} \rightarrow \omega a_{0}^{+}\right) \simeq \mathcal{B}\left(D_{s}^{+} \rightarrow \pi^{+} \pi^{0}\right)<$ $3.4 \times 10^{-4}$. In our calculation, $\mathcal{B}\left(D_{s}^{+} \rightarrow \rho^{0}\left(a_{0}^{+} \rightarrow\right.\right.$ $\left.\eta \pi^{+}\right)=\left(1.6_{-0.3}^{+0.2} \pm 0.6\right) \times 10^{-3}$ agrees with the data, whereas $\mathcal{B}\left(D_{s}^{+} \rightarrow \rho^{+}\left(a_{0}^{0} \rightarrow\right) K^{+} K^{-}\right)$is 10 times smaller than the observation, which requires a careful examination.
\end{abstract}

\section{Introduction}

The two-body $D_{s}^{+} \rightarrow P P, P V$ decays with $P(V)$ denoting the strangeless pesudoscalar (vector) meson have no configurations from the $W$-boson emission processes because $\bar{s}$ in $D_{s}^{+}$cannot be eliminated, as drawn in Fig. 1 for the topological diagrams $\mathrm{T}$ and $\mathrm{C}$. Interestingly, it leads to a specific exploration for the annihilation mechanism applied to $D_{s}^{+} \rightarrow \pi^{+} \pi^{0}, \pi^{+} \rho^{0}, \pi^{+} \omega[1-6]$.

In the short-distance $W$-boson annihilation (WA) $D_{s}^{+}(c \bar{s})$ $\rightarrow W^{+} \rightarrow u \bar{d}$ process, $u \bar{d}$ can be seen to move in the opposite directions in the $D_{s}^{+}$rest frame, such that there exists no orbital angular momentum between them. It indicates that $G(u \bar{d})=G\left(\pi^{+}\right)=+1$ with $G$ denoting the $G$ parity symmetry [3]. Since $G$-parity is a multiplicative quantum number, one obtains $G\left(\pi^{+} \rho^{0}, \pi^{+} \pi^{0}\right)=(+1,-1)$. Consequently, the WA $D_{s}^{+} \rightarrow \pi^{+} \rho^{0}\left(\pi^{+} \pi^{0}\right)$ decay due to $G(u \bar{d})=G\left(\pi^{+} \rho^{0}\right)\left[=-G\left(\pi^{+} \pi^{0}\right)\right]$ is a $G$-parity conserved (violated) process, which corresponds to the exper-

\footnotetext{
a e-mail: yuyao@cqupt.edu.cn

be-mail: yukuohsiao@gmail.com (corresponding author)

c e-mail: baiciank@ihep.ac.cn
}

imental result $\mathcal{B}\left(D_{s}^{+} \rightarrow \pi^{+} \rho^{0}\right)=(1.9 \pm 1.2) \times 10^{-4}$ $\left[\mathcal{B}\left(D_{s}^{+} \rightarrow \pi^{+} \pi^{0}\right)<3.4 \times 10^{-4}\right]$ [7]. By contrast, although the WA $D_{s}^{+} \rightarrow \pi^{+} \omega$ decay violates the $G$-parity symmetry, $\mathcal{B}\left(D_{s}^{+} \rightarrow \pi^{+} \omega\right)=(1.9 \pm 0.3) \times 10^{-3}$ shows no suppression [7]. It is hence considered to receive the long-distance annihilation contribution [1,3].

The $D_{s}^{+} \rightarrow S P, S V$ decays can help to investigate the short and long-distance annihilation mechanisms [8-10], where $S$ stands for a non-strange scalar meson. For example, the WA process for $D_{s}^{+} \rightarrow a_{0}^{0(+)} \pi^{+(0)}$ violates $G$ parity $[9,11]$, such that its branching fraction is expected as small as $\mathcal{B}\left(D_{s}^{+} \rightarrow \pi^{+} \pi^{0}\right)$. Nonetheless, one measures that $\mathcal{B}\left(D_{s}^{+} \rightarrow a_{0}^{0(+)} \pi^{+(0)}\right) \simeq 100 \mathcal{B}\left(D_{s}^{+} \rightarrow \pi^{+} \rho^{0}\right)$ $[7,12]$. Clearly, it indicates the main contribution from the long-distance annihilation process [9]. Explicitly, the longdistance annihilation process for $D_{s}^{+} \rightarrow a_{0}^{0(+)} \pi^{+(0)}$ starts with the $D_{s}^{+} \rightarrow \eta^{(\prime)} \rho^{+}$weak decay, followed by the $\eta^{(\prime)}$ and $\rho^{+}$rescattering. With the $\pi^{+(0)}$ exchange, $\eta^{(\prime)}$ and $\pi^{+}$ are turned into $a_{0}^{+(0)}$ and $\pi^{0(+)}$, respectively. Since BESIII has recently reported the first observation of the branching fractions of $D_{s}^{+} \rightarrow S V, S \rightarrow P P$ as $[13,14]$

$$
\begin{aligned}
\mathcal{B}_{+}\left(D_{s}^{+} \rightarrow a_{0}^{+} \rho^{0}, a_{0}^{+} \rightarrow \pi^{+} \eta\right) & =(2.1 \pm 0.8 \pm 0.5) \times 10^{-3}, \\
\mathcal{B}_{0}\left(D_{s}^{+} \rightarrow a_{0}^{0} \rho^{+}, a_{0}^{0} \rightarrow K^{+} K^{-}\right) & =(0.7 \pm 0.2 \pm 0.1) \times 10^{-3},
\end{aligned}
$$

we are wondering which of the short and long-distance annihilation processes can be the dominant contribution. Hence, we propose to study $D_{s}^{+} \rightarrow a_{0}^{+(0)} \rho^{0(+)}, D_{s}^{+} \rightarrow a_{0}^{+} \omega$, and the resonant three-body $D_{s}^{+} \rightarrow a_{0}^{+(0)} \rho^{0(+)}, a_{0}^{+(0)} \rightarrow$ $\left[\eta \pi^{+(0)}, K^{+} \bar{K}^{0}\left(K^{+} K^{-}\right)\right]$decays, in order to analyze the data in Eq. (1). We will also test if $\mathcal{B}\left(D_{s}^{+} \rightarrow a_{0}^{+} \rho^{0}, a_{0}^{+} \rho^{0}\right)$ have nearly equal sizes as $\mathcal{B}\left(D_{s}^{+} \rightarrow a_{0}^{+} \pi^{0}\right) \simeq \mathcal{B}\left(D_{s}^{+} \rightarrow\right.$ $a_{0}^{0} \pi^{+}$) that respects the isospin symmetry. 
Fig. 1 Topological diagrams for the Cabibbo-allowed $D_{s}^{+}$ weak decays

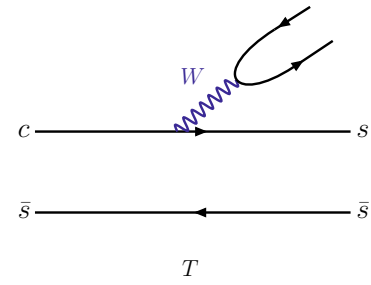

\section{Formalism}

Considering the short-distance WA processes, $D_{s}^{+} \rightarrow$ $P P(P V)$ and $D_{s}^{+} \rightarrow S P(S V)$ both get an $A$ term as the annihilation amplitude in Fig. 1. According to $\mathcal{B}\left(D_{s}^{+} \rightarrow\right.$ $\left.\pi^{+} \rho^{0}\right) \simeq 10^{-4}$ that receives the short-distance WA contribution [7], one regards $A$ to give $\mathcal{B}\left(D_{s}^{+} \rightarrow a_{0}^{+(0)} \rho^{0(+)}\right)$ not larger than $10^{-4}$. However, $\mathcal{B}_{+}$in Eq. (1) suggests $\mathcal{B}\left(D_{s}^{+} \rightarrow a_{0}^{+} \rho^{0}\right) \simeq 10^{-3}$. This strongly suggests that the main contribution to $D_{s}^{+} \rightarrow a_{0}^{+(0)} \rho^{0(+)}$ is from the longdistance annihilation process. For $D_{s}^{+} \rightarrow a_{0}^{+} \omega$, the WA contribution is suppressed with the $G$-parity violation, such that $A \simeq 0$. Therefore, we start with the triangle rescattering processes for $D_{s}^{+} \rightarrow a_{0}^{+(0)} \rho^{0(+)}$ and $D_{s}^{+} \rightarrow a_{0}^{+} \omega$ as the most possible main contributions.

See Fig. 2, the rescattering processes for $D_{s}^{+} \rightarrow a_{0}^{+(0)}$ $\rho^{0(+)}$ include both the weak and strong decays. In our case, the weak decays come from $D_{s}^{+} \rightarrow \pi^{+} \eta^{(\prime)}, K^{+} \bar{K}^{0}$, and the amplitudes are given by $[2-4,6]$
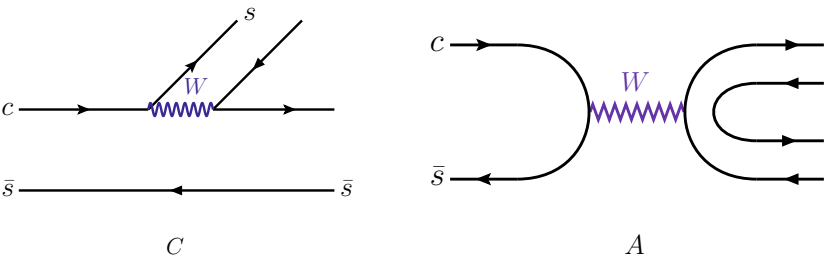

A

For the strong decays $V, S \rightarrow P P$, the amplitudes are given by [9]

$$
\begin{aligned}
& \mathcal{M}_{\rho^{0(+)} \rightarrow \pi^{+(0)} \pi^{-(+)}}=g_{\rho} \epsilon \cdot\left(q_{1}-q_{2}\right), \\
& \mathcal{M}_{a_{0}^{+} \rightarrow \eta^{(\prime)} \pi^{+}, K^{+} \bar{K}^{0}}=g_{\eta^{(\prime)}}, g_{K},
\end{aligned}
$$

where $\epsilon_{\mu}$ is the polarization four vector of the $\rho$ meson, and $q_{1,2}^{\mu}$ are the four momenta of $\pi^{+} \pi^{-}\left(\pi^{0} \pi^{+}\right)$, respectively. The $S U$ (3) flavor symmetry is able to relate different $V(S) \rightarrow P P$ decay channels $[17,18]$, such that we obtain $g_{\rho} / 2$ for $\mathcal{M}_{\rho^{0} \rightarrow K^{+} K^{-}, \bar{K}^{0} K^{0}}, g_{\rho} / \sqrt{2}$ for $\mathcal{M}_{\rho^{+} \rightarrow K^{+} \bar{K}^{0}}$, and $(-) g_{K} / \sqrt{2}$ for $\mathcal{M}_{a_{0}^{0} \rightarrow K^{+} K^{-}\left(\bar{K}^{0} K^{0}\right)}$, together with $(-) g_{\rho} / 2$ for $\mathcal{M}_{\omega \rightarrow K^{+} K^{-}\left(\bar{K}^{0} K^{0}\right)}$.

By assembling the weak and strong couplings in the rescattering processes, we derive that

$$
\begin{aligned}
& \mathcal{M}\left(D_{s}^{+} \rightarrow \rho^{0(+)} a_{0}^{+(0)}\right)=\mathcal{M}_{a}+\mathcal{M}_{a}^{\prime}+\mathcal{M}_{b}+\mathcal{M}_{c}, \\
& \mathcal{M}\left(D_{s}^{+} \rightarrow \omega a_{0}^{+}\right)=\hat{\mathcal{M}}_{b}+\hat{\mathcal{M}}_{c},
\end{aligned}
$$

with $M_{a}^{(\prime)}$ and $M_{b(c)}\left[\hat{M}_{b(c)}\right]$ for Fig. 2a, b(c), respectively. More explicitly, $\mathcal{M}_{a, b, c}$ are given by $[9,19,20]$

$$
\begin{aligned}
\mathcal{M}_{a} & =\int \frac{d^{4} q_{1}}{(2 \pi)^{4}} \frac{\mathcal{M}_{\eta} \mathcal{M}_{\rho^{0(+)} \rightarrow \pi^{+} \pi^{-(0)}} \mathcal{M}_{a_{0}^{+(0)} \rightarrow \eta \pi^{+(0)}} F_{\pi}\left(q_{2}^{2}\right)}{\left(q_{1}^{2}-m_{\pi}^{2}+i \epsilon\right)\left[\left(q_{1}-p_{2}\right)^{2}-m_{\pi}^{2}+i \epsilon\right]\left[\left(q_{1}-p_{1}\right)^{2}-m_{\eta}^{2}+i \epsilon\right]}, \\
\mathcal{M}_{b} & =\int \frac{d^{4} q_{1}}{(2 \pi)^{4}} \frac{\mathcal{M}_{K} \mathcal{M}_{\rho^{0(+)} \rightarrow K^{+} K^{-}\left(K^{+} \bar{K}^{0}\right)} \mathcal{M}_{a_{0}^{+(0)} \rightarrow \bar{K}^{0} K^{+(0)}} F_{K}\left(q_{2}^{2}\right)}{\left(q_{1}^{2}-m_{K}^{2}+i \epsilon\right)\left[\left(q_{1}-p_{2}\right)^{2}-m_{K}^{2}+i \epsilon\right]\left[\left(q_{1}-p_{1}\right)^{2}-m_{K}^{2}+i \epsilon\right]}, \\
\mathcal{M}_{c} & =\int \frac{d^{4} q_{1}}{(2 \pi)^{4}} \frac{\mathcal{M}_{K} \mathcal{M}_{\rho^{0(+)} \rightarrow \bar{K}^{0} K^{0(+)}} \mathcal{M}_{a_{0}^{+(0)} \rightarrow \bar{K}^{0} K^{+(0)}} F_{K}\left(q_{2}^{2}\right)}{\left(q_{1}^{2}-m_{K}^{2}+i \epsilon\right)\left[\left(q_{1}-p_{2}\right)^{2}-m_{K}^{2}+i \epsilon\right]\left[\left(q_{1}-p_{1}\right)^{2}-m_{K}^{2}+i \epsilon\right]},
\end{aligned}
$$

$$
\begin{aligned}
\mathcal{M}_{\eta}\left(D_{s}^{+} \rightarrow \pi^{+} \eta\right) & =\frac{G_{F}}{\sqrt{2}} V_{c s}^{*} V_{u d}(\sqrt{2} A \cos \phi-T \sin \phi), \\
\mathcal{M}_{\eta^{\prime}}\left(D_{s}^{+} \rightarrow \pi^{+} \eta^{\prime}\right) & =\frac{G_{F}}{\sqrt{2}} V_{c s}^{*} V_{u d}(\sqrt{2} A \sin \phi+T \cos \phi), \\
\mathcal{M}_{K}\left(D_{s}^{+} \rightarrow K^{+} \bar{K}^{0}\right) & =\frac{G_{F}}{\sqrt{2}} V_{c s}^{*} V_{u d}(C+A),
\end{aligned}
$$

where $G_{F}$ is the Fermi constant, $V_{c S}^{*} V_{u d}$ the CabibboKobayashi-Maskawa (CKM) matrix elements, and $V_{c s}^{*} V_{u d} \simeq$ 1 presents the Cabibbo-allowed decay modes. In addition, $(T, C, A)$ are the topological amplitudes, along with the mixing angle $\phi=43.5^{\circ}$ from the $\eta-\eta^{\prime}$ mixing matrix $[15,16]$ :

$$
\left(\begin{array}{c}
\eta \\
\eta^{\prime}
\end{array}\right)=\left(\begin{array}{cc}
\cos \phi & -\sin \phi \\
\sin \phi & \cos \phi
\end{array}\right)\left(\begin{array}{c}
\sqrt{1 / 2}(u \bar{u}+d \bar{d}) \\
s \bar{s}
\end{array}\right) .
$$

with $q_{2}=q_{1}-p_{2}$ and $q_{3}=q_{1}-p_{1}$ following the momentum flows in Fig. 2. The form factor $F_{M}\left(q_{2}^{2}\right) \equiv$ $\left(\Lambda_{M}^{2}-m_{M}^{2}\right) /\left(\Lambda_{M}^{2}-q_{2}^{2}\right)$ with the cutoff parameter $\Lambda_{M}$ $[M=(\pi, K)]$ is to avoid the overestimation with $q_{2}$ to $\pm \infty$ [21]. Substituting $\eta^{\prime}$ and $\omega$ for $\eta$ in $\mathcal{M}_{a}$ and $\rho^{0}$ in $\mathcal{M}_{b(c)}$ leads to $\mathcal{M}_{a}^{\prime}$ and $\hat{\mathcal{M}}_{b(c)}$, respectively.

As a consequence, we find that

$$
\begin{aligned}
\mathcal{M}\left(D_{s}^{+} \rightarrow \rho^{0} a_{0}^{+}\right) & =\mathcal{M}\left(D_{s}^{+} \rightarrow \rho^{+} a_{0}^{0}\right), \\
\mathcal{M}\left(D_{s}^{+} \rightarrow \omega a_{0}^{+}\right) & =0,
\end{aligned}
$$

where the first relation respects the isospin symmetry, whereas $\hat{\mathcal{M}}_{b}=-\hat{\mathcal{M}}_{c}$ due to $\mathcal{M}_{\omega \rightarrow K^{+} K^{-}}=-\mathcal{M}_{\omega \rightarrow \bar{K}^{0} K^{0}}$ cancels the rescattering contributions to $D_{s}^{+} \rightarrow \omega a_{0}^{+}$, which causes $\mathcal{M}\left(D_{s}^{+} \rightarrow \omega a_{0}^{+}\right)=0$. 
Fig. 2 Triangle rescattering diagrams for $D_{s}^{+} \rightarrow \rho^{0(+)} a_{0}^{+(0)}$ and $D_{s}^{+} \rightarrow \omega a_{0}^{+}$

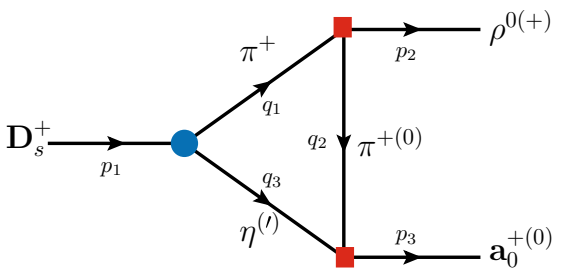

(a)

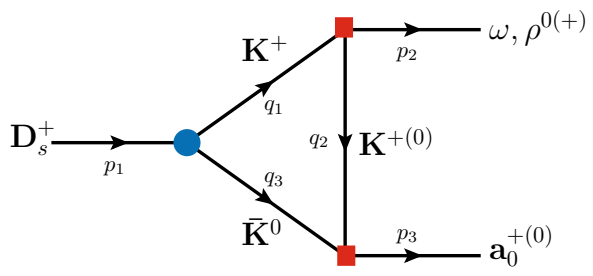

(b)

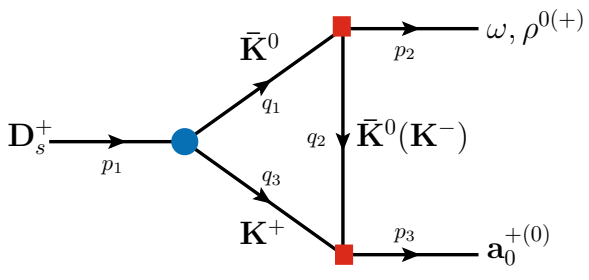

(c)
To deal with the triangle loops in Eq. (6), the equation in Refs. [22-25] can be useful, given by
In the above equation, $1 / D_{a_{0}}(s)$ presents the propagator for $a_{0}$, and we define [26]

$$
\begin{aligned}
& \int \frac{d^{4} q_{1}}{i \pi^{2}} \frac{q_{1}^{\mu}}{\left(q_{1}^{2}-m_{1}^{2}+i \epsilon\right)\left[\left(q_{1}-p_{2}\right)^{2}-m_{2}^{2}+i \epsilon\right]\left[\left(q_{1}-p_{1}\right)^{2}-m_{3}^{2}+i \epsilon\right]} \\
& =-p_{\rho}^{\mu} C_{1}\left(p_{a_{0}}^{2}, m_{\rho}^{2}, m_{D_{s}}^{2}, m_{1}^{2}, m_{2}^{2}, m_{3}^{2}\right)-p_{D_{s}}^{\mu} C_{2}\left(p_{a_{0}}^{2}, m_{\rho}^{2}, m_{D_{s}}^{2}, m_{1}^{2}, m_{2}^{2}, m_{3}^{2}\right)
\end{aligned}
$$

By calculating the triangle rescattering processes, we obtain

$$
\begin{aligned}
& \Gamma\left(D_{s}^{+} \rightarrow \rho^{0+} a_{0}^{+(0)}\right)=\frac{\left|\mathbf{p}_{2}\right|^{3}}{8 \pi m_{\rho}^{2}}|H|^{2}, \\
& H=\frac{1}{8 \pi^{2}}\left(g_{\rho} g_{\eta} \mathcal{M}_{\eta} C_{\eta}+g_{\rho} g_{\eta^{\prime}} \mathcal{M}_{\eta^{\prime}} C_{\eta^{\prime}}+g_{\rho} g_{K} \mathcal{M}_{K} C_{K}\right),
\end{aligned}
$$

with $C_{\eta^{(\prime)}}=C_{2, \eta^{(\prime)}}-C_{2, \eta^{(\prime)}}^{*}$ and $C_{K}=C_{2, K}-C_{2, K}^{*}$ as the integrated results of the $C_{2}$ terms in Eq. (8), where $C_{2, M}^{(*)}$ are defined by

$$
\begin{aligned}
C_{2, \eta}^{(*)} & =C_{2}\left(m_{\pi}, m_{\pi}\left(\Lambda_{\pi}\right), m_{\eta}\right), \\
C_{2, \eta^{\prime}}^{(*)} & =C_{2}\left(m_{\pi}, m_{\pi}\left(\Lambda_{\pi}\right), m_{\eta^{\prime}}\right), \\
C_{2, K}^{(*)} & =C_{2}\left(m_{K}, m_{K}\left(\Lambda_{K}\right), m_{K}\right) .
\end{aligned}
$$

However, the $C_{1}$ terms have been disappearing due to $p_{\rho}^{\mu} \cdot \epsilon=$ 0 in the amplitudes.

For the three-body decays $D_{s}^{+} \rightarrow \rho^{0(+)}\left(a_{0}^{+(0)} \rightarrow\right) \eta \pi^{+(0)}$, $K^{+} \bar{K}^{0}\left(K^{+} K^{-}\right)$, we present [7]

$\Gamma\left(D_{s}^{+} \rightarrow \rho a_{0}, a_{0} \rightarrow \eta \pi(K K)\right)$

$$
=\iint \frac{1}{(2 \pi)^{3}} \frac{\left|\mathbf{p}_{2}\right|^{2}}{32 m_{D_{s}} m_{\rho}^{2}}|H|^{2} \frac{g_{\eta(K)}^{2}}{\left|D_{a_{0}}(s)\right|^{2}} d s d t,
$$

with $s=\left(p_{\eta(K)}+p_{\pi(K)}\right)^{2}$ and $t=\left(p_{\rho}+p_{\pi(K)}\right)^{2}$, such that the theoretical results can be compared to the data in Eq. (1).

$$
D_{a_{0}}(s)=s-m_{a_{0}}^{2}-\sum_{\alpha \beta}\left[\operatorname{Re} \Pi_{a_{0}}^{\alpha \beta}\left(m_{a_{0}}^{2}\right)-\Pi_{a_{0}}^{\alpha \beta}(s)\right],
$$

where

$$
\begin{aligned}
\Pi_{a_{0}}^{\alpha \beta}(x)= & \frac{G_{\alpha \beta}^{2}}{16 \pi}\left\{\frac{m_{\alpha \beta}^{+} m_{\alpha \beta}^{-}}{\pi x} \log \left[\frac{m_{\beta}}{m_{\alpha}}\right]-\theta\left[x-\left(m_{\alpha \beta}^{+}\right)^{2}\right]\right. \\
& \times \rho_{\alpha \beta}\left(i+\frac{1}{\pi} \log \left[\frac{\sqrt{x-\left(m_{\alpha \beta}^{+}\right)^{2}}+\sqrt{x-\left(m_{\alpha \beta}^{-}\right)^{2}}}{\sqrt{x-\left(m_{\alpha \beta}^{-}\right)^{2}}-\sqrt{x-\left(m_{\alpha \beta}^{+}\right)^{2}}}\right]\right) \\
& -\rho_{\alpha \beta}\left(1-\frac{2}{\pi} \arctan \left[\frac{\sqrt{-x+\left(m_{\alpha \beta}^{+}\right)^{2}}}{\sqrt{x-\left(m_{\alpha \beta}^{-}\right)^{2}}}\right]\right) \\
& \times\left(\theta\left[x-\left(m_{\alpha \beta}^{-}\right)^{2}\right]-\theta\left[x-\left(m_{\alpha \beta}^{+}\right)^{2}\right]\right) \\
+ & \rho_{\alpha \beta} \frac{1}{\pi} \log \left[\frac{\sqrt{\left(m_{\alpha \beta}^{+}\right)^{2}-x}+\sqrt{\left(m_{\alpha \beta}^{-}\right)^{2}-x}}{\sqrt{\left(m_{\alpha \beta}^{-}\right)^{2}-x}-\sqrt{\left(m_{\alpha \beta}^{+}\right)^{2}-x}}\right] \\
& \left.\times \theta\left[\left(m_{\alpha \beta}^{-}\right)^{2}-x\right]\right\}, \\
\rho_{\alpha \beta} \equiv & \sqrt{x-\left(m_{\alpha \beta}^{+}\right)^{2}} \sqrt{x-\left(m_{\alpha \beta}^{-}\right)^{2}} \mid / x,
\end{aligned}
$$

with $G_{\alpha \beta}=\left(g_{\eta^{(\prime)}}, g_{K}\right)$ for $\alpha \beta=\left(\eta^{(\prime)} \pi, K \bar{K}\right)$ and $m_{\alpha \beta}^{ \pm}=$ $m_{\alpha} \pm m_{\beta}$. 


\section{Numerical results}

In the numerical analysis, we adopt $V_{c s}=V_{u d}=1-\lambda^{2} / 2$ with $\lambda=0.22453 \pm 0.00044$ in the Wolfenstein parameterization [7], along with $m_{a_{0}^{0}}=0.987 \mathrm{GeV}[27,28]$. The topological parameters $(T, C, A)$ have been extracted as [6]

$$
\begin{aligned}
(|T|,|C|,|A|)= & (0.363 \pm 0.001,0.323 \pm 0.030,0.064 \\
& \pm 0.004) \mathrm{GeV}^{3} \\
\left(\delta_{C}, \delta_{A}\right)= & \left(-151.3 \pm 0.3,23.0_{-10.0}^{+} 7.0\right.
\end{aligned}
$$

where $\delta_{C, A}$ are the relative strong phases. According to the extraction, we obtain $\mathcal{B}\left(D_{s}^{+} \rightarrow \pi^{+} \eta, \pi^{+} \eta^{\prime}, K^{+} \bar{K}^{0}\right)=$ $(1.8 \pm 0.2,4.2 \pm 0.5,3.1 \pm 0.5) \times 10^{-2}$, consistent with the experimental values of $(1.68 \pm 0.10,3.94 \pm 0.25,2.95 \pm$ $0.14) \times 10^{-2}$, respectively [7]. For $V, S \rightarrow P P$, the strong coupling constants read $[7,27,28]$

$$
\begin{gathered}
g_{\rho}=6.0,\left(g_{\eta}, g_{\eta^{\prime}}, g_{K}\right)=(2.87 \pm 0.09,-2.52 \\
\pm 0.08,2.94 \pm 0.13) \mathrm{GeV}
\end{gathered}
$$

Empirically, $\Lambda_{M}$ of $\mathcal{O}(1.0 \mathrm{GeV})$ is commonly used to explain the data [29-31]; besides, it is obtained that $\Lambda_{K}-\Lambda_{\pi}=$ $m_{K}-m_{\pi}$ [32]. Therefore, we are allowed to use $\left(\Lambda_{\pi}, \Lambda_{K}\right)=$ $(1.25 \pm 0.25,1.60 \pm 0.25) \mathrm{GeV}$, which result in

$$
\begin{aligned}
C_{\eta} & =[(0.57 \pm 0.08)+i(0.17 \pm 0.09)] \mathrm{GeV}^{-2}, \\
C_{\eta^{\prime}} & =[(0.34 \pm 0.03)-i(0.27 \pm 0.07)] \mathrm{GeV}^{-2}, \\
C_{K} & =[(0.85 \pm 0.03)-i(0.45 \pm 0.08)] \mathrm{GeV}^{-2},
\end{aligned}
$$

with $p_{a_{0}}^{2}=m_{a_{0}}^{2}$. Subsequently, we predict

$$
\begin{aligned}
& \mathcal{B}\left(D_{s}^{+} \rightarrow \rho^{0(+)} a_{0}^{+(0)}\right)=(3.0 \pm 0.3 \pm 1.0) \times 10^{-3}, \\
& \mathcal{B}\left(D_{s}^{+} \rightarrow \omega a_{0}^{+}\right)=0,
\end{aligned}
$$

where the first error in $\mathcal{B}\left(D_{s}^{+} \rightarrow \rho^{0(+)} a_{0}^{+(0)}\right)$ takes into account the uncertainties from $\Lambda_{\pi}$ and $\Lambda_{K}$, and the second one combines those from $V_{c s}^{*}, V_{u d},(T, C, A)$, and the strong coupling constants. For the resonant three-body decays, we obtain

$$
\begin{aligned}
& \mathcal{B}\left(D_{s}^{+} \rightarrow \rho^{0(+)}\left(a_{0}^{+(0)} \rightarrow\right) \eta \pi^{+(0)}\right) \\
& \quad=\left(1.6_{-0.3}^{+0.2} \pm 0.6\right) \times 10^{-3}, \\
& \mathcal{B}\left(D_{s}^{+} \rightarrow \rho^{+}\left(a_{0}^{0} \rightarrow\right) K^{+} K^{-}, K^{0} \bar{K}^{0}\right) \\
& \quad=\left(0.9_{-0.1}^{+0.1} \pm 0.4,0.7_{-0.1}^{+0.1} \pm 0.3\right) \times 10^{-4}, \\
& \mathcal{B}\left(D_{s}^{+} \rightarrow \rho^{0}\left(a_{0}^{+} \rightarrow\right) K^{+} \bar{K}^{0}\right) \\
& \quad=\left(1.5_{-0.3}^{+0.2} \pm 0.6\right) \times 10^{-4},
\end{aligned}
$$

where the sources of the two errors are the same as those in Eq. (17).

\section{Discussions and conclusions}

In the triangle loop, when the momentum flow approaches the mass shell for one of the three propagators, the integration with $i \epsilon \simeq i m \Gamma$ gives rise to the imaginary parts in Eq. (16), where $\Gamma$ is a very tiny decay width for $\pi, \eta^{(\prime)}$ or $K$. The off-shell integrations are responsible for the real parts in Eq. (16), for which we take $\mathcal{M}_{a}$ as our description. In principle, the integration allows a momentum flow from $-\infty$ to $+\infty$. However, when the exchange particle proceeds with $q_{2}^{2}$ around several $\mathrm{GeV}^{2}$, instead of the infinity, the integration with $q_{2}^{2} \sim \pm \infty$ causes an overestimation [21]. We have accordingly introduced the form factor $F_{\pi}$ in Eq. (6) to cut off the contribution from $q_{2}^{2} \sim \pm \infty$. While $q_{1}^{2}, q_{2}^{2}$ and $q_{3}^{2}$ have been associated in the loop, $F_{\pi}$ also works to cut off the contributions from the propagators of the rescattering particles $\pi^{+}$and $\eta^{(\prime)}$. Note that the single cutoff form factor has also been commonly used elsewhere [33-35]. ${ }^{1}$

The smallness of the WA $D_{s}^{+} \rightarrow M M$ decay can be traced back to its amplitude, given by [37-39]

$$
\begin{aligned}
& \mathcal{M}_{W A}\left(D_{s}^{+} \rightarrow M M\right) \propto f_{D_{s}} q^{\mu}\left\langle M M\left|\bar{u} \gamma_{\mu}\left(1-\gamma_{5}\right) d\right| 0\right\rangle \\
& \quad \simeq\left(m_{u}+m_{d}\right)\left\langle M M\left|\bar{u} \gamma_{5} d\right| 0\right\rangle,
\end{aligned}
$$

where $q^{\mu}\left\langle M M\left|u \gamma_{\mu} d\right| 0\right\rangle=0$ corresponds to the conservation of the vector current (CVC); most importantly, $m_{u(d)} \simeq$ 0 causes the chiral suppression of the WA $D_{s}^{+} \rightarrow M M$ decay. According to the data, $\mathcal{B}\left(D_{s}^{+} \rightarrow \pi^{+} \rho^{0}\right)=(1.9 \pm 1.2) \times 10^{-4}$ indicates that $\mathcal{B}_{W A}\left(D_{s}^{+} \rightarrow M M\right)$ should be around $10^{-4}$. In addition, $\mathcal{B}\left(D_{s}^{+} \rightarrow \pi^{+} \pi^{0}\right)<3.4 \times 10^{-4}$ suggests that the G-parity violation suppresses the WA process even more. Therefore, since $D_{s}^{+} \rightarrow \rho a_{0}, \rho \pi$ are both the Gparity conserved processes, it is reasonable to present that $\mathcal{B}_{W A}\left(D_{s}^{+} \rightarrow \rho a_{0}\right) \simeq \mathcal{B}_{W A}\left(D_{s}^{+} \rightarrow \rho \pi\right) \sim 10^{-4}$. As a theoretical support, we present

$$
\begin{aligned}
& \mathcal{B}_{W A}\left(D_{s}^{+}(c \bar{s}) \rightarrow \rho a_{0}\right) \\
& \quad=R_{f} \frac{\left(V_{c s}^{*} V_{u d} f_{D_{s}}\right)^{2} \tau_{D_{s}}}{\left(V_{c b} V_{u d}^{*} f_{B_{c}}\right)^{2} \tau_{B_{c}}} \mathcal{B}_{W A}\left(B_{c}^{+}(c \bar{b}) \rightarrow \rho a_{0}\right) \sim 10^{-4},
\end{aligned}
$$

in agreement with our estimation, where $R_{f}=3.1 \times 10^{-2}$ is mostly from the phase space factors, and $\mathcal{B}_{W A}\left(B_{c}^{+} \rightarrow\right.$ $\left.\rho a_{0}\right) \simeq 1.0 \times 10^{-5}$ is adopted from Ref. [40].

Disregarding the WA contributions, we predict $\mathcal{B}\left(D_{s}^{+} \rightarrow\right.$ $\left.\rho^{0(+)} a_{0}^{+(0)}\right)=3.0 \times 10^{-3}$ in Eq. (17). It is found that the $\eta \pi, \eta^{\prime} \pi, K^{+} \bar{K}^{0}$ rescatterings and their interferences give $6 \%, 7 \%, 30 \%$ and $59 \%$ of $\mathcal{B}\left(D_{s}^{+} \rightarrow a_{0}^{+(0)} \rho^{0(+)}\right)$, respectively. By contrast, the $\rho \eta^{(\prime)}$ rescatterings from $D_{s}^{+} \rightarrow \rho \eta^{(\prime)}$ dominantly contribute to $D_{s}^{+} \rightarrow a_{0}^{+(0)} \pi^{0(+)}$, instead of

${ }_{1}^{1}$ Please also consult Refs. [36], where one considers three cutoff form factors. 
$D_{s}^{+} \rightarrow K^{*} K$ [9]. Since $D_{s}^{+} \rightarrow \omega a_{0}^{+}$has no rescattering effects; besides, the WA contribution is suppressed by the $G$-parity violation, we anticipate that $\mathcal{B}\left(D_{s}^{+} \rightarrow \omega a_{0}^{+}\right) \simeq$ $\mathcal{B}\left(D_{s}^{+} \rightarrow \pi^{+} \pi^{0}\right)<3.4 \times 10^{-4}[7]$.

In Eq. (18), $\mathcal{B}\left(D_{s}^{+} \rightarrow \rho^{0}\left(a_{0}^{+} \rightarrow\right) \eta \pi^{+}\right)=\left(1.6_{-0.3}^{+0.2} \pm\right.$ $0.6) \times 10^{-3}$ is able to explain the data [see Eq. (1)], demonstrating the sufficient long-distance annihilation contribution. It is confusing that $\mathcal{B}\left(D_{s}^{+} \rightarrow a_{0}^{0} \rho^{+}, a_{0}^{0} \rightarrow K^{+} K^{-}\right)$ is 10 times smaller than $\mathcal{B}_{0}$ in Eq. (1). For clarification, we take the approximate form of the resonant branching fraction: $\mathcal{B}\left(D_{s}^{+} \rightarrow S V, S \rightarrow P P\right) \simeq \mathcal{B}\left(D_{s}^{+} \rightarrow S V\right) \mathcal{B}(S \rightarrow P P)$, together with the isospin relation: $\mathcal{B}\left(D_{s}^{+} \rightarrow \rho^{0} a_{0}^{+}\right) \simeq$ $\mathcal{B}\left(D_{s}^{+} \rightarrow \rho^{+} a_{0}^{0}\right)$, such that $\mathcal{B}_{0} / \mathcal{B}_{+}$is reduced as $\mathcal{B}\left(a_{0}^{0} \rightarrow\right.$ $\left.K^{+} K^{-}\right) / \mathcal{B}\left(a_{0}^{+} \rightarrow \eta \pi^{+}\right) \simeq 1 / 3$, disagreeing with $\mathcal{B}\left(a_{0}^{0} \rightarrow\right.$ $\left.K^{+} K^{-}\right) / \mathcal{B}\left(a_{0}^{+} \rightarrow \eta \pi^{+}\right)=1 / 10$ from the experimental extraction [8]. Therefore, we conclude that there exists a possible contradiction between the observations in Eq. (1).

In our reasoning, the contradiction might be caused by $D_{s}^{+} \rightarrow \rho^{+} f_{0}, f_{0} \rightarrow K^{+} K^{-}$with $f_{0} \equiv f_{0}(980)$, which can be mistaken as $D_{s}^{+} \rightarrow \rho^{+} a_{0}^{0}, a_{0}^{0} \rightarrow K^{+} K^{-}$[41]. First, since $D_{s}^{+} \rightarrow \rho^{+} f_{0}$ is an external $W$-boson emission process, its branching fraction can be of order $10^{-3}$. Second, $a_{0}$ and $f_{0}$ are both scalar mesons, and have nearly the same masses and overlapped decay widths. As a result, it is possible that one cannot distinguish between the resonant signals of $D_{s}^{+} \rightarrow \rho^{+}\left(f_{0}, a_{0}\right),\left(f_{0}, a_{0}\right) \rightarrow K^{+} K^{-}$in the $K^{+} K^{-}$invariant mass spectrum. For a careful examination, we suggest a measurement of $\mathcal{B}\left(D_{s}^{+} \rightarrow \rho^{0}\left(a_{0}^{+} \rightarrow\right.\right.$ ) $\left.K^{+} \bar{K}^{0}\right) / \mathcal{B}\left(D_{s}^{+} \rightarrow \rho^{+}\left(a_{0}^{0} \rightarrow\right) K^{+} K^{-}\right)$, which will be observed around 2 if there exists no resonant $f_{0} \rightarrow K^{+} K^{-}$ decay to be involved in $\mathcal{B}\left(D_{s}^{+} \rightarrow \rho^{+} K^{+} K^{-}\right)$.

In summary, we have studied $D_{s}^{+} \rightarrow \rho^{0(+)} a_{0}^{+(0)}, D_{s}^{+} \rightarrow$ $\omega a_{0}^{0}$, and the resonant $D_{s}^{+} \rightarrow \rho a_{0}, a_{0} \rightarrow \eta \pi(K K)$ decays. In the final state interaction, where $D_{s}^{+} \rightarrow \eta^{(\prime)} \pi^{+}\left(K^{+} \bar{K}^{0}\right)$ is followed by the $\eta^{(\prime)} \pi^{+}\left(K^{+} \bar{K}^{0}\right)$ to $\rho a_{0}$ rescattering, we have predicted $\mathcal{B}\left(D_{s}^{+} \rightarrow \rho^{0(+)} a_{0}^{+(0)}\right)=(3.0 \pm 0.3 \pm 1.0) \times 10^{-3}$. Because of the non-contribution of the rescattering effects and the suppressed short-distance $W$ annihilation, it has been expected that $\mathcal{B}\left(D_{s}^{+} \rightarrow \omega a_{0}^{+}\right) \simeq \mathcal{B}\left(D_{s}^{+} \rightarrow \pi^{+} \pi^{0}\right)<$ $3.4 \times 10^{-4}$. For the resonant three-body decay, $\mathcal{B}\left(D_{s}^{+} \rightarrow\right.$ $\left.\rho^{0}\left(a_{0}^{+} \rightarrow\right) \eta \pi^{+}\right)=\left(1.6_{-0.3}^{+0.2} \pm 0.6\right) \times 10^{-3}$ has been shown to agree with the data. We have presented $\mathcal{B}\left(D_{s}^{+} \rightarrow \rho^{+}\left(a_{0}^{0} \rightarrow\right.\right.$ ) $\left.K^{+} K^{-}\right) 10$ times smaller than the observation, indicating that a more careful examination is needed.

\footnotetext{
Acknowledgements We would like to thank Prof. Liang Sun for useful discussions. YKH was supported in part by NSFC (Grant nos. 11675030 and 12175128). YY was supported in part by NSFC (Grant nos. 11905023 and 12047564), the Fundamental Research Funds for the Central Universities (Grant no. 2020CDJQY-Z003) and CQCSTC (Grant nos. cstc2020jcyj-msxmX0555 and cstc2020jcyjmsxmX0810). BCK was supported in part by NSFC (Grant no. 118750 54 ) and the Chinese Academy of Sciences (CAS) Large-scale Scien-
}

tific Facility Program; Joint Large-Scale Scientific Facility Fund of the NSFC and CAS (Contract no. U2032104).

Data Availability Statement This manuscript has no associated data or the data will not be deposited. [Authors' comment: The data used in this paper are publicly available and they can be found in the corresponding references.]

Open Access This article is licensed under a Creative Commons Attribution 4.0 International License, which permits use, sharing, adaptation, distribution and reproduction in any medium or format, as long as you give appropriate credit to the original author(s) and the source, provide a link to the Creative Commons licence, and indicate if changes were made. The images or other third party material in this article are included in the article's Creative Commons licence, unless indicated otherwise in a credit line to the material. If material is not included in the article's Creative Commons licence and your intended use is not permitted by statutory regulation or exceeds the permitted use, you will need to obtain permission directly from the copyright holder. To view a copy of this licence, visit http://creativecomm ons.org/licenses/by/4.0/.

Funded by $\mathrm{SCOAP}^{3}$.

\section{References}

1. S. Fajfer, A. Prapotnik, P. Singer, J. Zupan, Phys. Rev. D 68, 094012 (2003)

2. B. Bhattacharya, J.L. Rosner, Phys. Rev. D 81, 014026 (2010)

3. H.Y. Cheng, C.W. Chiang, Phys. Rev. D 81, 074021 (2010)

4. F.S. Yu, X.X. Wang, C.D. Lu, Phys. Rev. D 84, 074019 (2011)

5. H.N. Li, C.D. Lu, Q. Qin, F.S. Yu, Phys. Rev. D 89, 054006 (2014)

6. H.Y. Cheng, C.W. Chiang, Phys. Rev. D 100, 093002 (2019)

7. P.A. Zyla et al., [Particle Data Group], PTEP. 2020, 083 C01 (2020)

8. H.Y. Cheng, C.W. Chiang, Phys. Rev. D 81, 074031 (2010)

9. Y.K. Hsiao, Y. Yu, B.C. Ke, Eur. Phys. J. C 80, 895 (2020)

10. X.Z. Ling, M.Z. Liu, J.X. Lu, L.S. Geng, J.J. Xie, Phys. Rev. D 103, $116016(2021)$

11. N.N. Achasov, G.N. Shestakov, Phys. Rev. D 96, 036013 (2017)

12. M. Ablikim et al., BESIII, Phys. Rev. Lett. 123, 112001 (2019)

13. M. Ablikim et al., BESIII, Phys. Rev. D 104, 071101 (2021)

14. M. Ablikim et al., BESIII, Phys. Rev. D 104, 032011 (2021)

15. T. Feldmann, P. Kroll, B. Stech, Phys. Rev. D 58, 114006 (1998)

16. T. Feldmann, P. Kroll, B. Stech, Phys. Lett. B 449, 339 (1999)

17. N.A. Tornqvist, Ann. Phys. 123, 1 (1979)

18. Fayyazuddin and Riazuddin, A Modern Introduction To Particle Physics, 3rd edn. (World Scientific, Singapore, 2011)

19. Y. Yu, Y.K. Hsiao, Phys. Lett. B 820, 136586 (2021)

20. Y.K. Hsiao, Y. Yu, Phys. Rev. D 104, 034008 (2021)

21. M.C. Du, Q. Zhao, Phys. Rev. D 104, 036008 (2021)

22. G.T. Hooft, M.J.G. Veltman, Nucl. Phys. B 153, 365 (1979)

23. G. Passarino, M.J.G. Veltman, Nucl. Phys. B 160, 151 (1979)

24. T. Hahn, M. Perez-Victoria, Comput. Phys. Commun. 118, 153 (1999)

25. A. Denner, S. Dittmaier, Nucl. Phys. B 734, 62 (2006)

26. N.N. Achasov, A.V. Kiselev, Phys. Rev. D 70, 111901 (2004)

27. M. Ablikim et al., BESIII, Phys. Rev. D 95, 032002 (2017)

28. D.V. Bugg, Phys. Rev. D 78, 074023 (2008)

29. N.A. Tornqvist, Z. Phys. C 61, 525 (1994)

30. X.Q. Li, D.V. Bugg, B.S. Zou, Phys. Rev. D 55, 1421 (1997)

31. Q. Wu, D. Y. Chen, X.J. Fan, G. Li, Eur. Phys. J. C 79, 265 (2019)

32. H.Y. Cheng, C.K. Chua, A. Soni, Phys. Rev. D 71, 014030 (2005)

33. Q. Wu, D.Y. Chen, T. Matsuki, Eur. Phys. J. C 81, 193 (2021)

34. Q. Wu, D.Y. Chen, Phys. Rev. D 100, 114002 (2019)

35. G. Li, X.H. Liu, Z. Zhou, Phys. Rev. D 90, 054006 (2014) 
36. Y. Cheng, Q. Zhao, arXiv:2106.12483 [hep-ph]

37. Y.K. Hsiao, C.Q. Geng, Phys. Rev. D 91, 077501 (2015)

38. Y.K. Hsiao, S.Y. Tsai, C.C. Lih, E. Rodrigues, JHEP 04, 035 (2020)

39. X. Huang, Y.K. Hsiao, J. Wang, L. Sun, arXiv:2109.02897 [hep-ph]
40. X. Liu, Z.J. Xiao, Phys. Rev. D 82, 054029 (2010)

41. Private communications with the group members at BESIII 